Revue d'histoire de l'Amérique française

REVUE D.HISTOIRE DE L'AMÉRIQUE FRANÇAISE

\title{
Le mariage sous le régime français (suite)
}

\section{Paul-André Leclerc}

Volume 14, numéro 1, juin 1960

URI : https://id.erudit.org/iderudit/302029ar

DOI : https://doi.org/10.7202/302029ar

Aller au sommaire du numéro

Éditeur(s)

Institut d'histoire de l'Amérique française

ISSN

0035-2357 (imprimé)

1492-1383 (numérique)

Découvrir la revue

Citer cet article

Leclerc, P.-A. (1960). Le mariage sous le régime français (suite). Revue d'histoire de l'Amérique française, 14(1), 34-60. https://doi.org/10.7202/302029ar d'utilisation que vous pouvez consulter en ligne.

https://apropos.erudit.org/fr/usagers/politique-dutilisation/ 


\title{
LE MARIAGE SOUS LE RÉGIME FRANÇAIS *
}

\author{
(suite) \\ Chaprtre III \\ CAS SPECIAUX
}

Jusqu'ici nous avons parlé des mariages qui se sont célébrés dans les conditions ordinaires; il nous reste à considérer les cas spéciaux et les exceptions aux règles générales. Plusieurs circonstances peuvent se greffer autour de la célébration d'un mariage; elles ne sont pas toutes extraordinaires, mais nous avons préféré étudier rapidement les circonstances ordinaires entourant un mariage, quitte à revenir brièvement sur ce qui nous semble présenter un intérêt particulier. Avant de passer à la célébration populaire du mariage, c'est-à-dire les noces, nous allons jeter un rapide coup d'œil sur la légitimation des enfants, la bénédiction du lit nuptial, le nombre de mariages, les quêtes au mariage et les mariages avec des sauvagesses; nous nous arrêterons quelque peu sur les mariages à la gaumine et les secondes noces, les filles du roi, les dots et les contrats de mariage.

Les enfants nés en dehors du légitime mariage ne furent pas nombreux sous le Régime français; il est facile de contrôler puisque ce fait devait être noté dans les registres; c'est ainsi que Ferland peut conclure:

Dans l'espace de soixante-neuf ans, (16211690), au milieu d'une population composée de militaires, de marins, de voyageurs, de nouveaux colons, deux enfants seulement sont nés hors du légitime mariage de leurs parents. Ces chiffres fournissent une réfutation péremptoire des calomnies inventées par les La Hontan et quelques aventuriers de même aloi, contre la réputation de nos aỉeules canadiennes. ${ }^{45}$

* Voir notre Revue, XIII : 230-246, 374-401, 525-543.

45 Ferland, op. cit., 40. 
Un autre cas prévu par le Rituel et probablement un peu plus fréquent, c'est celui de la légitimation par un mariage subséquent. Monseigneur de Saint-Vallier prescrit la ligne de conduite à suivre dans cette situation:

Lorsque les Mariez auront eû des enfants avant leur Mariage, qu'ils voudront faire légitimer, on les mettra dans un endroit particulier sous le voile avec l'Epoux \& l'Epouse, \& le Prêtre dira ... ${ }^{64}$

Suit une longue oraison demandant la miséricorde divine pour enlever cette tache et permettre aux coupables de réparer. Le Rituel ajoute:

S'il y a eu des enfans avant le Mariage, qui ayent été légitimez en la Celebration du Mariage, on l'exprimera, \& l'on marquera leur nom, leur âge, $\&$ le lieu où ils auront été baptisez. ${ }^{47}$

Nous avons rencontré un cas de légitimation par mariage subséquent dans les registres de Sainte Foy, le 15 janvier 1760:

Et (avons) légitimé un garçon nommé ( ) constansinot que le dit ignace Constansinot déclare et reconnait être son légitime enfant né de la ditte marie Joseph Doucet avant le mariage contracté.48

Quant à l'expression placer sous le voile, nous n'avons pas trouvé de détails; la cérémonie consistait sans doute à placer un voile symbolique sur la tête des époux et de leur enfant à légitimer, pendant que le prêtre récitait une oraison spéciale; ceci se faisait dans un endroit particulier, c'est-à-dire retiré. Cependant cette légitimation devait se faire après le mariage, puisque le fait était noté dans le registre, probablement à la sacristie, ou au presbytère, avant la signature des registres.

La bénédiction du lit nuptial est facultative, mais si les nouveaux époux la désirent, il faut suivre certaines prescriptions:

Si des nouveaux Mariez demandent qu'on benisse leur Lit, Nous ordonnons en ce cas que la Benediction s'en fasse apres la Celebration du Ma-

$46 \mathrm{Mgr}$ de Saint-Vallier, op. cit., 358.

47 Ibid., 645.

48 Registres de la Paroisse Notre-Dame de Foy, 15 janvier 1760. 
riage avant le Dîner; afin que la modestie y soit gardée de telle maniere, que rien ne s'y fasse contre la sainteté de cette Ceremonie. ${ }^{49}$

Ces précautions prises, le curé adresse encore une belle exhortation, non seulement aux nouveaux époux, mais aussi à tous les assistants :

Nous ne pouvons nous dispenser de vous dire avec S. Paul, qu'il est necessaire que le Mariage soit traité de tous avec honnêteté, \& que le Lit Nuptial doit être pur \& sans tache; vous souvenant que vous êtes les enfans des Saints \& de Dieu même: Que vôtre chair par l'union du Verbe avec la nature humaine, est devenuë la chair de J.C. Que vos corps sont le Temple du S. Esprit, que vous n'y devez toucher que comme à des Vases Sacrez; c'est-à-dire avec modestie \& pudeur. Souvenez-vous que vôtre Lit Nuptial sera un jour le lit de vôtre mort, d'où vos ames seront enlevées pour être presentées au Tribunal de Dieu, pour y recevoir le terrible châtiment des sept Maris de Sara, si vous vous y rendez comme eux esclave de vôtre chair, de vos passions, $\&$ de vôtre concupiscence. Joignez vos Prieres aux nôtres, \& demandez à Dieu qu'il détourne de vous un sort si malheureux, qu'il éloigne de vôtre Lit \& de vos cœurs l'esprit d'impureté, \& qu'il y fasse regner celui de la chasteté. ${ }^{50}$

Après ces paroles, tous les assistants se mettent à genoux et le curé procède alors à la bénédiction du lit nuptial et de ceux qui sont présents. Le prêtre récite une oraison:

Visita, quæsumus, Domine, habitationem istam, $\&$ omnes insidias Diaboli ab ea longe repelle, Angeli tui sancti habitent in ea, qui vos in pace custodiant, $\&$ benedictio Domini sit super vos semper. Per Christum Dominum nostrum. R. Amen. ${ }^{51}$

(Visitez, nous vous en prions, Seigneur, cette maison, et repoussez loin d'elle toutes les embûches du Démon, que vos saints Anges l'habitent, qu'ils vous gardent en paix, et que la bénédiction du Seigneur soit toujours sur vous. Par le Christ NotreSeigneur. Ainsi soit-il.)

49 Mgr de Saint-Vallier, op. cit., 359.

50 Loc. cit.

51 Ibid., 360. 
Cette oraison finie, le curé récite le psaume 127 suivi d'une douzaine de versets et du «Pater Noster »; une nouvelle oraison termine ces prières:

Oremus. Benedic Domine thalamum hunc, una cum his conjugibus; ut in tua pace consistant, \& in tua voluntate permaneant, tuo amore vivant \& senescant, \& multiplicentur in longitudinem dierum, $\&$ ad regna coelorum perveniant. Per Christum Dominum nostrum. R. Amen. Benedicat vos Pater, \& Filius, \& Spiritus Sanctus: \& cor vestrum sinceri, sancti, ac casti amoris copulet nexu perpetuo. $R$. Amen. ${ }^{52}$

(Prions, Bénissez Seigneur ce lit nuptial, ainsi que ces conjoints, pour qu'ils restent fermes dans votre paix, qu'ils demeurent dans votre volonté, qu'ils vivent et vieillissent dans votre amour, qu'ils se multiplient pendant la durée de leur vie et qu'ils parviennent au règne du ciel. Par le Christ NotreSeigneur. Ainsi soit-il. Que vous bénissent le Père, le Fils et l'Esprit Saint; que votre cœur s'unisse par le lien perpétuel d'un amour sincère, chaste et saint. Ainsi soit-il.)

La cérémonie se termine par l'aspersion de l'eau bénite sur le lit, sur les nouveaux mariés et aussi sur tous ceux qui sont présents. Ces prières de l'Église sont un gage de bonheur pour les nouveaux époux et renferment des conseils précieux pour la conduite de leur vie conjugale.

Sous le Régime français, nous pouvons dire que les mariages ont été nombreux puisque le chiffre officiel est de 25,464 mariages pour les années 1608 à $1760 .{ }^{53}$ Cependant le nombre de mariages pendant les premières décades est plutôt restreint; en effet, de 1608 à 1700 , il n'y eut que 3,913 mariages. ${ }^{54}$ Durant les décades qui suivirent, les mariages se multiplièrent régulièrement, de sorte qu'en 1730 le total des mariages se chiffre à $10,405 .{ }^{55}$ Ce nombre passe à 14,074 en $1740,{ }^{56}$ puis à 19,031 en

52 Loc. cit.

53 Tanguay, op. cit., 179.

54 Ibid., 85.

55 Ibid., 128.

56 Ibid., 140. 
$1750,{ }^{57}$ pour atteindre le chiffre donné plus haut à la fin du Régime français.

Il semble bien que la coutume de faire une quête au mariage n'était pas généralisée; toutefois, nous avons trouvé un cas où il est fait mention que les quêtes aux mariages ont rapporté «14fr. 10s. » pour l'année 1717 à Beauport. ${ }^{58}$ Cette année-là, six mariages avaient été célébrés dans cette paroisse.

Nous avons parlé jusqu'ici des mariages célébrés régulièrement; cependant il s'est introduit même en notre pays une coutume un peu extraordinaire, les mariages appelés à la Gaumine. Un décret du Concile de Trente se lit comme suit:

A l'égard de ceux qui entreprendront de se marier autrement qu'en la presence de leur Curé, ou Prêtre qui ait la permission, ou celle de l'Ordinaire $\&$ en la presence de deux ou trois Témoins; le Saint Concile les rend tout-à-fait inhabiles à contracter de cette manière, \& déclare les Mariages qui se seront faits ainsi, nuls \& invalides, comme dés à present par ce Decret il les casse \& annulle.59

Les prescriptions du Concile de Trente étaient donc claires et précises: pas de mariage possible sans la présence du curé et de deux ou trois témoins. Cependant, en France, on chercha un moyen d'échapper à cette législation; voici comment les faits se passèrent:

Un certain Gaumin se présenta devant son curé avec sa promise et deux témoins. Les deux amants exprimèrent devant le curé et les témoins leur consentement matrimonial et s'en retournèrent chez eux. Ils prétendirent ensuite qu'ils avaient satisfait au décret «Tametsi » et qu'ils étaient régulièrement mariés. De là, le nom de mariage à la gaumine. Ceux qui suivirent Gaumin allongèrent encore la corde. Ils entraient dans l'église pendant une messe quelconque, se mettaient dans le même banc puis au moment de l'Elévation se donnaient la main, devant les deux témoins qu'ils avaient amenés. Le mariage était bâclé sans autre formalité. ${ }^{60}$

57 Ibid., 150.

58 Langevin, op. cit., 95.

59 Mgr de Saint-Vallier, op. cit., 343s.

60 Les Cahiers des Dix, IV : 87. 
La législation civile édicta des peines contre les contractants et leurs complices; cette coutume regrettable gagna notre pays dès les débuts du dix-huitième siècle. En 1711, à Beauport, se produisait un incident de ce genre:

Le mariage à la gaumine qui fit sans contredit le plus de bruit dans le pays fut celui de Louis de Montéléon, officier dans les troupes du détachement de la marine, avec Mlle de l'Estringant de SaintMartin. Les jeunes étourdis se marièrent ainsi dans l'église de Beauport, le 7 janvier 1711. Ce mariage fut réhabilité le 16 février suivant. A cause de la haute situation des deux familles concernées, cette équipée occupa la chronique scandaleuse pendant plusieurs semaines. ${ }^{61}$

Nous comprenons facilement que cette aventure ait fait les frais des conversations durant une bonne période de temps, puisque le mariage ne fut réhabilité qu'un mois et quelques jours plus tard ainsi qu'il est noté dans les registres de Beauport:

J'ai donné la bénédiction nuptiale et en qualité de Prestre faisant les fonctions curiales célébré le mariage entre Louis Ecuier Sieur de Montéléon fils de desfunt Paul Ecuier Sieur de Montéléon Bouteiller du Roy de la ville de Paris d'une part et Damoiselle Marie Anne Joseph De L'Estringuent fille de Alexandre Joseph De L'Estringuent Ecuier Sieur de St. Martin et de Dame Magdelene Louise Juchereau de cette paroisse de Beauport. ${ }^{62}$

Le procès fait à l'occasion de ce mariage fut assez compliqué puisqu'il couvre quarante pages du Rapport de l'Archiviste. ${ }^{63}$ Le 24 mai 1717, Monseigneur de Saint-Vallier frappe un grand coup pour empêcher que ces choses pénibles ne se reproduisent:

Ayant égard à la requête de notre promoteur, et aux autres remontrances qui nous ont été faites, nous déclarons excommuniés, d'une excommunication encourue par le seul fait, et dont nous nous réservons à nous seul le pouvoir d'absoudre, tous ceux qui dans la suite oseront contracter de si détestables mariages,

61 Pierre-Georges Roy, Les Petites Choses de Notre Histoire, I: 185.

62 Langevin, op. cit., 99.

63 RAPQ (1920-1921) : 368-407. 
soit devant leur curé, soit devant d'autres prêtres, soit séculiers, soit réguliers; ceux qui seront assez méchants pour le conseiller, tous les témoins apostés pour les dits mariages, et les notaires qui en dresseraient l'acte, sauf à notre official, dans les cas particuliers qui seront portés devant lui, d'imposer encore d'autres peines particulières pour punir les prévaricateurs. ${ }^{64}$

Après ce mandement de Monseigneur de Saint-Vallier, il se produisit encore quelques cas de mariages à la gaumine; ils nous sont rapportés par les registres. Le 23 décembre 1727, un mariage à la gaumine se produisit à Batiscan:

Pendant que le curé Gervais Lefebvre, de Batiscan, célébrait une messe basse, le sieur Daniel Portail, de Gevron, fils de Daniel Portail, maire perpétuel de la ville de Saint-Florent-le-Vieil, diocèse d'Angers, contractait mariage avec Marie-Anne Antoinette Langy de Levrard, et ce, à l'insçue du curé. Les coupables firent réhabiliter leur mariage le 8 septembre de l'année suivante. ${ }^{65}$

Ce mariage s'est fait après le Mandement de Monseigneur de Saint-Vallier: les contractants et leurs témoins furent donc excommuniés par le fait même. Le 28 février 1724, un autre mariage de cette espèce avait été tenté à Boucherville:

On célèbre à Boucherville le mariage de Jean Desnoyers et Marie-Thérèse Ménard, lesquels s'étaient mariés à la Gomine, quelques années auparavant, pendant que leur missionnaire, M. de Francheville, célébrait la messe. ${ }^{66}$

Le décret d'excommunication porté par Monseigneur de Saint-Vallier a sans aucun doute porté fruit en diminuant de beaucoup le nombre de mariages à la gaumine. Le 15 février 1754, le fait se présente encore, cette fois à la Pointe-aux-Trembles de Montréal, tel qu'inscrit dans les registres:

Les nommés Pierre Benard et Catherine Laviolette, s'étant mariés, le 15 f'évrier, à la gomine, pendant l'élévation de la Sainte-Hostie, le Mandement de $\mathrm{M}^{\mathrm{gr}}$ de Saint-Valier, contre ces détestables

64 Mandements des Evêques de Québec, I : 493s.

65 Tanguay, op. cit., 124.

66 Ibid., 119. 
mariages, a été lu au prône, le dimanche d'après, $24^{\text {me }}$ jour du même mois, sur l'Ordonnance de Mr. Lenormant, Vicaire-Général du diocèse, et les prétendus mariés ont été déclarés, en conséquence, excommuniés. ${ }^{67}$

Une autre tentative de mariage à la gaumine fut manquée, si l'on peut dire, à cause de la surdité du prêtre qui disait alors sa messe:

Le 9 février 1751, le nommé BarthélémyJoseph Richard, maître d'école, à Québec, âgé de 43 ans, et la veuve Divertissans se rendaient à la cathédrale de Québec et là, pendant une messe basse dite par M. de la Villangevin, au moment de l'élévation, déclaraient hautement qu'ils se prenaient pour mari et femme. M. de la Villangevin quelque peu sourd n'entendit pas les paroles prononcées par Richard et la veuve Divertissans. Mais les personnes présentes rapportèrent la chose au curé Récher qui fit arrêter le maître d'école Richard, Celui-ci, devant la Prévôté, nia s'être marié à la gaumine. Il fut acquitté sous le prétexte qu'il s'était rendu à l'église avec la veuve Divertissans simplement à l'effet de lui faire une promesse de mariage. ${ }^{68}$

Comme nous pouvons le constater, les mariages à la gaumine ne furent pas tellement nombreux sous le Régime français; le décret d'excommunication porté par Monseigneur de SaintVallier y est pour beaucoup, car notre peuple était profondément chrétien; dans tous les cas les coupables se repentirent et revinrent à l'Église.

Une autre difficulté qui se présentait dans la préparation des mariages, c'était la preuve de liberté quand il s'agissait d'étrangers ou de veufs. C'est pourquoi Monseigneur de SaintVallier donne à ses curés des directives précises:

Nous sommes bien-aises de faire remarquer ici aux Curez l'obligation étroite où ils sont de ne pas remarier facilement ceux \& celles de leurs Paroisses qui demanderoient de l'être, sous pretextes que leurs maris, ou leurs femmes seroient absens depuis quel-

67 Ibid., 156.

68 P.-G. Roy, op. cit., I: 186. 
ques années, \& qu'ils assureroient être morts à cause de cette longue absence. La regle qu'ils doivent garder est de ne pas écouter ce qu'on voudra leur dire sur cela, jusqu'à ce qu'on leur fasse representer un Certificat en bonne forme, qui atteste la mort de la personne. Pour peu qu'il y ait sujet de douter, Nous desirons qu'ils ne passent pas outre, \& qu'ils s'adressent à Nous pour sçavoir ce qu'ils auront à faire dans ces occasions. Ils garderont la même conduite à l'égard des Vagabonds, Sodats \& Etrangers qui voudroient se marier. ${ }^{69}$

Pour les étrangers, les curés doivent être encore plus prudents :

Si les personnes sont Veuves \& d'un autre Diocese, le Certificat du Curé, qui atteste la mort de l'une des deux Parties, doit être legalisé par la signature de l'Evêque Diocésain, \& ensuite reconnu de Nous, ou de nos Grands-Vicaires, avant qu'on puisse passer outre à la celebration du Mariage. ${ }^{70}$

Telles sont les exigences du Rituel, mais avant cela, dès 1691 , Monseigneur de Saint-Vallier avait donné des directives:

L'expérience ayant fait voir qu'il se trouve des personnes venues de France qui demandent à se marier au Canada, sans qu'elles puissent prouver qu'elles n'ont point contracté mariage en d'autres lieux, ou que la personne avec qui elles l'ont contracté soit morte; nous voulons pour obvier aux inconvénients qui pourraient arriver, que les personnes ci-dessus ne soient point reçues au Sacrement de Mariage qu'elles ne produisent des Certificats légalisés et en forme, venus de France, ou autres témoignages assurés, approuvés de Nous ou de nos Grands Vicaires, qu'ils ne sont point actuellement mariés. ${ }^{71}$

Avant de partir pour la France, l'évêque de Québec fait encore ses recommandations :

Nous voudrions que vous inspirassiez à ceux qui viennent de France et qui ont désir de se marier, de faire venir des attestations légalisées pour prouver

$69 \mathrm{Mgr}$ de Saint-Vallier, op. cit., 335.

70 Ibid., 340.

71 Mandements des Evêques de Québec, I: 277. 
qu'ils ne sont pas mariés. Vous les instruirez de la préparation qu'ils doivent apporter à la réception de ce Sacrement; rien n'étant plus déplorable que l'état et la disposition où se trouvent la plupart des gens qui contractent mariage. ${ }^{22}$

Dans le second synode, tenu à Ville-Marie en 1694, les mêmes exigences sont répétées :

Ils (les curés) ne sauraient être trop exacts à demander aux étrangers et inconnus, avant de les marier, des preuves certaines qu'ils n'ont point contracté avec d'autres; et afin de n'y être point trompés, Nous ordonnons dans les cas particuliers et embarrassants qu'ils Nous consultent, ou nos Grands Vicaires. ${ }^{73}$

De même dans le quatrième synode de Québec tenu en 1700 , nous pouvons lire:

Ils avertiront qu'ils ont reçu un ordre particulier de Nous, de ne point marier les personnes qui l'ont déjà été, à moins qu'ils n'apportent une Attestation en bonne forme de la mort de leurs femmes ou de leurs maris. ${ }^{74}$

Enfin dans une ordonnance particulière pour le règlement de son diocèse, Monseigneur de Saint-Vallier ajoute:

Les Curés prendront garde de marier ceux qui sont nouvellement établis dans l'étendue de leurs Paroisses, à moins qu'ils n'y aient fait un séjour de six mois. Ils auront soin de prendre les précautions absolument nécessaires pour savoir s'ils ne sont pas mariés en France, ou en d'autres Paroisses de ce Diocèse. ${ }^{75}$

Les ordonnances sont claires et précises: avant de marier des veufs ou des étrangers, les curés doivent exiger des témoignages de liberté. Ces preuves peuvent se présenter sous plusieurs formes selon les circonstances et la facilité littéraire des signataires. Nous en trouvons de nombreux exemples cités dans le Rapport de l'Archiviste pour les années 1951-1953. Nous ne

72 Ibid., 285.

73 Ibid., 319.

74 Ibid., 397.

75 Ibid., 412. 
citons que quelques témoignages de liberté; c'est suffisant, croyons-nous, pour nous donner une idée juste de ce procédé:

Mathurin Fillion natif de Breu en Bretagne domestique chez $\mathrm{mr}$ fontbonne Commandant de guyenne a obtenu permission de faire publier ses bans de mariage sur le certificat signé Maliene $C^{\text {pne }}$ du regiment Royal Comtois par lequel Il le declare Libre de mariage. En date du $148^{\text {bre }} 1752$ et du certificat de mr. fontbonne par lequel il déclare qu'il est à son service depuis 4 ans et demi et que depuis ce tems il scait qu'il ne s'est point marié. Briand Ptre sec. ${ }^{76}$

Les dits jour et an que dessus est comparu devant nous martin Lapits charpentier natif de sarre eveché de Bayonne age de 30 ans depuis 7 en Canada Lequel après nous avoir promis de nous dire La verité nous a assuré que Le dit Michel daguerre n'est point marié en france et ce pour le connoittre des l'enfance, étant de la même $\mathrm{p}^{\text {sse }}$ pour avoir appris le metier ensemble à St Jean de Luz être parti ensemble de bayonne et venu en Canada 1750 par le même $\mathrm{v}^{\text {au }}$ Et Lecture faitte a declaré Le tout véritable et ne scavoir signer Briand ptre, sec. ${ }^{77}$

Nous missionair de charlebourg certifions que Jean Baptiste chenay dit clairmont soldat de La compagnie de $\mathrm{Mr}$ de Lanaudiere est libre suivant Les connoissance par Ecrit que nous en avons eu de sa mere A Quebec le 9 août 1757 Morisseaux Ptre.

Sur ce nous avons permis au dit chenay de faire publier ses bans, à Québec le $9^{\mathrm{e}}$ aoust 1757 Briand Ptre sec. ${ }^{78}$

Les dits jour et an que dessus est comparu Jean L'espagnol demeurant à Quebec Lequel a affirmé par serment que le dit Collar n'est point marié $1^{\mathrm{e}}$ parce qu'il le retire chez soy depuis plus de sept ans $2^{\mathrm{e}}$ parce qu'il etoit très jeune et petit quand il est venu chez soy et a declaré ne scavoir signer Briand Ptre sec. ${ }^{78 a}$

76 RAPQ (1951-1953) : 5.

77 Ibid., 8.

78 Ibid., 14

78a Ibid., 15. 
Guille Lacroix de Coutance $\mathrm{p}^{\mathrm{sse}} \mathrm{du}$ taon a obtenu permission de se marier sur les depositions de julien veval et de Jean mallet Recües par mons ${ }^{\text {gr }}$ qui m'a ordonné d'en faire mention sur les registres Le 15e Xbre 1757. Briand Ptre sec. ${ }^{78 b}$

Un témoignage de liberté qui prend un intérêt historique particulier, c'est celui qui permet à la veuve d'Alexis Gagné dit Belavance de se remarier; en effet, Alexis Gagné faisait partie du groupe de deux cent cinquante prisonniers, pris par les Anglais à la bataille des Plaines d'Abraham; l'histoire ne nous dit pas quel fut le traitement réservé à ces hommes, mais nous avons des détails intéressants dans ce témoignage de liberté:

Nous, Alexis Dumontier, demeurant à la Pointeà-Lacaille (Saint-Thomas), certifions en notre âme et conscience ce qui suit: 1er. - Qu'après avoir été faits prisonniers à Québec, le 13 septembre 1759, avec quantité d'autres Canadiens, nous avons été mis dans un transport tous ensemble quelques jours, et qu'après, nous avons été divisés pour être mis dans d'autres bâtiments.

2e. - Que tous les Canadiens prisonniers ont été passés en revue deux ou trois fois devant que de passer en Angleterre, et qu'après les dites revues, ils ont été tous embarqués sur des vaisseaux de guerre et conduit à Plimouth.

3e. - Qu'étant arrivés à Plimouth, on nous a fait tous débarquer et conduire en prison où nous sommes restés quatre mois, et, après les dits quatre mois, nous avons été conduits à Dieppe, port de France.

4e. - Qu'étant arrivés à Dieppe avec tous les prisonniers, M. Lacombière-Lacorne m'a chargé de faire une liste de tous les prisonniers canadiens, que j'ai faite très exactement sans en omettre aucun.

5e. - Que, Alexis Gagné dit Belavance, de la paroisse de Saint-Pierre, Rivière-du-Sud, mon parent n'a pas paru parmi les prisonniers, dans les revues qui ont été faites en Canada, devant que d'embarquer sur les vaisseaux pour aller à Plimouth, ni en arrivant à Plimouth, ni à Dieppe quand $M$.

78b Ibid., 35. 
Lacombière-Lacorne a fait faire la liste de tous les prisonniers canadiens, ce qui est une preuve qu'il est mort dans le combat, comme je le pense. Alexis Dumontier, 23 février $1764 .^{79}$

Il est certainement arrivé quelques fois qu'un témoignage de liberté soit erroné; nous avons le cas de Pierre Picher qui a épousé Catherine Durand, le 25 novembre 1665 ; de fait sa première femme, appelée Lefebvre, était encore vivante. C'est pourquoi, le onze septembre 1673, le Conseil Souverain fut saisi de la requête suivante:

La requeste de Pierre Picher contenant qu'estant en ce pays en 1642, Louis Picher, son frère, luy avait envoyé une lettre missive, par laquelle il luy mandait que Marie Lefebvre, sa femme, estait décédée: sur lequel advis estant sorty de son engagement, il se serait marié avec Catherine Durand, duquel mariage sont issus trois enfants (...) depuis laquelle lettre le suppliant n'avait receu aucune nouvelle de ses parents, ny de ceux de la dicte défuncte Lefebvre jusqu'en 1671, qu'un homme venant de France luy dist qu'elle estait encore vivante et que ce qui luy avait esté mandé n'estait pas véritable. (...) Ayant obtenu son congé, il passa en France, où il trouva la dicte Lefebvre encore vivante, recognaissant par là qu'il avait esté surpris par la diste lettre. Et comme il avait beaucoup de tendresse pour ses dicts trois enfants et pour la dicte Durand qu'il avait abusée par innocence, il s'était resolu de repasser en ce pays, où pour lors le dict sieur de Pétrée luy avait conseillé d'amener avec luy la dicte Lefebvre, ce qu'il avait faict. Et s'estant embarqué dans le navire La Nouvelle-France, commandé par le capitaine Poullet, la dicte Lefebvre serait décédée. Et comme il aurait esté arrivé en cette ville, il s'estait remis, par autorité de l'Eglise, avec la dicte Durand. (...) Le Conseil suppléant à défault de lettres de Chancellerie a légitimé les enfans issus du dict Picher et de la dicte Catherine Durand, et les a déclarés habiles à leur succéder. (...) Le grand vicaire du sieur Evesque de Pétrée, vicaire apostolique en ce pays, sera adverty par

${ }^{79}$ Cloutier, op. cit., II: $171 \mathrm{~s}$. 
le dict substitut d'attendre les décisions du Conseil à réhabiliter aucunes personnes par sacrement de mariage en matière semblable. Frontenac. ${ }^{80}$

La Providence s'était chargée de libérer le pauvre Picher de la situation embarrassante où il se trouvait placé ! Le 13 août 1726, il est noté dans les registres de la Pointe-aux-Trembles de Québec (Neuville), un cas de doute:

Charles Cauhet épousait en secondes noces à la Pointe-aux-Trembles de Québec, Marie-Charlotte Laroche. Ils avaient déjà contracté mariage, mais comme il s'était élevé un doute raisonnable au sujet de la mort de sa première femme, défense avait été faite à Marie-Charlotte Laroche, le 30 octobre 1725, d'habiter avec son prétendu mari, jusqu'à ce que preuve certaine de liberté fut produite par Cauhet. ${ }^{81}$

Le témoignage de liberté le plus simple, c'est le certificat de sépulture. Le 12 août 1748, un nommé Mathurin Chapelet épousait à Québec Angelique Demitre; seize ans plus tard, nous pouvons lire:

Dans les registres des Procès-verbaux de liberté, nous voyons qu'Angelique Demitre obtient la permission d'épouser Pierre Batz, le 4 juin 1764, après avoir donné la preuve que son mari Chapelet était mort à l'hôpital Saint-Pierre de la Martinique. ${ }^{82}$

Sous le Régime français, nous rencontrons beaucoup de mariages en secondes noces: les noyades étaient fréquentes, et la température, plutôt rude, apportait souvent des maladies, parfois mortelles. Nous avons trouvé quelques cas de troisièmes noces, de quatrièmes et même de cinquièmes noces. Mlle Eléonore de Granmaison qui a vécu soixante-dix ans, de 1622 à 1692, a épousé quatre maris: Antoine Boudier, sieur de Beauregard, François de Chavigny, sieur de Berchereau, Nicolas Gourdeau de Beaulieu et le sieur de La Tesserie. ${ }^{83}$

Une femme, nommée Anne Jousselot, a épousé cinq maris ; née en 1659, elle épousait Joseph Galois en 1677, Toussaint Du-

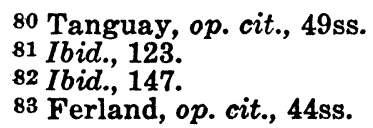


beau le 23 mai 1678, André Duval le 21 juillet 1698, Jean Maranda le 13 juin 1712, Claude Dubreuil le 11 octobre 1725; elle vécut encore dix-huit ans, puisqu'elle ne mourut qu'en 1743: selon le proverbe, l'appétit vient en mangeant! C'est la seule femme qui s'est mariée cinq fois. ${ }^{84}$

De même, il n'y a qu'un homme qui se soit marié cinq fois ; Tanguay rapporte le cas ainsi :

Jean-Baptiste Cauchon, cultivateur au Château-

Richer, épouse en cinquièmes noces, Louise Pinguet

dit Laglardière. (Reg. de Québec). ${ }^{85}$

Il arrivait parfois que le veuvage était réduit à sa plus simple expression; les circonstances poussaient les veuves à convoler en justes noces, car il fallait vivre et peupler la colonie. Le 12 février 1686, Jean Giroux se remariait quatre mois et demi après le décès de Marie Daufin, sa première femme. ${ }^{86}$ Le 3 août 1711, Jean Allard se remariait; il était «veuf du 23 avril $\gg .{ }^{87}$ Catherine Nau, veuve depuis deux mois seulement, épousait J. B. Peuvret, en juillet 1659.88 Le 28 janvier 1648, Françoise Morin, veuve d'Antoine Pelletier, se remariait à Etienne Dumay (Demers), après trois mois et demi de veuvage. ${ }^{89}$ Marie Morin, veuve de Jean Bouteiller dit Testu, épousait trois mois plus tard Thomas Letendre, le 3 janvier 1699.90 Comme nous le constatons, sous le Régime français, la période de veuvage était souvent abrégée, tant pour les hommes que pour les femmes.

Pour la célébration des secondes noces, il y a des rubriques spéciales à suivre et Monseigneur de Saint-Vallier les indique dans son Rituel:

Il n'y a rien de particulier à observer dans les secondes Nôces, sinon que quand c'est la Femme qui se marie pour la seconde fois, on ne bénit pas les nouveaux Mariéz avant la Communion, \& on ne les

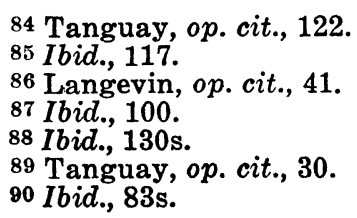


met pas sous le Voile; mais le Curé les fait approcher de l'Autel, aprés la Messe finie, pour dire sur eux les Prieres suivantes. ${ }^{91}$

Le curé récite alors les psaumes 127 et 126 qui chantent la joie du retour après l'exil et l'inutilité des efforts sans l'appui de Yahweh; une nombreuse postérité est un bienfait de Yahweh. Une douzaine de versets appellent ensuite le secours de Dieu et la cérémonie se termine par une oraison:

Oremus. Praetende, quaesumus Domine, fidelibus tuis dexteram coelestis auxilii; ut te toto corde perquirant, \& quae digne postulant assequi mereantur, Per Christum Dominum nostrum. R. Amen..$^{92}$

(Prions. Tendez, nous vous en prions Seigneur, à vos fidèles la main droite du secours céleste; afin qu'ils vous recherchent de tout leur cœur, et ce qu'ils demandent convenablement, qu'ils méritent de l'obtenir. Par le Christ Notre-Seigneur. Ainsi soitil.)

Le Rituel ajoute: destie. $^{93}$

Le Mari \& la Femme se retireront avec mo-

Après la cérémonie religieuse, on célèbre la fête comme pour les premières noces; nous reviendrons sur ce sujet un peu plus loin.

Qui de nous n'a entendu dire: les Canadiens français ont du sang sauvage dans les veines ? C'est pourquoi beaucoup de gens se sont inquiétés de nos origines; les badauds ont fait courir toutes sortes d'histoires plus ou moins abracadabrantes sur ce sujet et la légende s'est emparée de ces inventions pour les fixer dans les esprits. C'est pourquoi, nous allons brièvement jeter un coup d'œil sur l'origine de nos premières aïeules mariées au pays sous le Régime français.

Les mariages entre blancs et sauvagesses semblent avoir été très peu nombreux; le premier en date eut lieu le 3 novembre 1644:

$91 \mathrm{Mgr}$ de Saint-Vallier, op. cit., 361.

92 Ibid., 362.

93 Loc. cit. 
On célèbre le premier mariage d'un français avec une sauvagesse, béni par l'Eglise à Québec. Le marié s'appelait Martin Prevost et l'épouse MarieOlivier (Manitouabewich) ${ }^{\mathbf{9 4}}$

Ferland ajoute que Martin Prevost était originaire de «Montreuil-sur-le-Bois-de-Vincennes » et que le père de Marie Olivier s'appelait « Roch Manitouabeouich ${ }^{.95}$

Le mariage du genre qui est le plus célèbre est sans aucun doute celui de Pierre Boucher qui fut, à deux reprises, gouverneur des Trois-Rivières. Ferland rapporte ainsi ce mariage:

Le fils de Gaspar, Pierre Boucher épousa en 1649, la fille d'un chef sauvage, élevée par les Ursulines. Marie Chrestienne, ainsi se nommait-elle, avait reçu de l'instruction; sa signature apposée au contrat de mariage est d'une main ferme et nette; elle survécut peu de temps à son mariage.

Trois ans plus tard, en 1652, Pierre Boucher épousait une blanche, Jeanne, fille de Christophe Crevier. ${ }^{96}$

De fait les mariages entre blancs et sauvages furent-ils vraiment fréquents ? C'est Benjamin Sulte qui nous en donne la réponse:

De 1608 à 1663, il y a eu sept mariages de Français avec des Huronnes et des Alconquines, par permissions spéciales. Cinq de ces mariages ont laissé des enfants. De 1663 à 1673: on constate cinq mariages mixtes (entre blancs et sauvages) dans le cours de ces dix années; et quatre autres de 1674 à 1700. (Donc 16 mariages de 1608 à 1700). (...) Il ne nous est pas permis d'en accepter qu'un moindre nombre pour le dix-huitième (siècle), vu que les Sauvages diminuaient rapidement et que nous augmentions dans des proportions étonnantes.

Mais, dira-t-on, à part ces alliances reconnues par l'Eglise et l'Etat, il devait y en avoir à la mode des Sauvages. Nous le croyons, c'est probable. (...) Les enfants issus de ces rencontres ne pouvaient pas être Canadiens-français; ils ont dû suivre leurs mères dans les bois, car autrement nous

94 Tanguay, op. cit., 27.

95 Ferland, op. cit., 62.

96 Ibid., 61. 
les retrouverions chez-nous, vu que les registres disent tout ce qui s'est passé à l'égard des mariages. Ce furent les sources des métis, dont les descendants sont aujourd'hui des Sauvages. Au lieu d'avoir sous ce rapport emprunté au sang indigène, nous y avons plutôt mêlé le nôtre en pure perte. ${ }^{97}$

Un problème qui revêt une acuité particulière, c'est celui de l'émigration féminine vers notre pays au dix-septième siècle. Il existe une légende persistante contre la bonne réputation des demoiselles qui vinrent se marier ici au Canada. Cette émigration se divise en deux parties: jusqu'à 1663, puis les filles du roi de 1663 à $1673 .^{98}$

Les émigrantes de la première période sont venues en notre pays par initiative privée tandis que celles du deuxième groupe étaient recrutées et transportées par autorité royale, de là vient leur nom unique de filles du roi. La rumeur s'est vite accréditée que ces filles étaient toutes des Madeleines et qu'on les déportait afin d'en libérer la France! De telles affirmations étaient faciles à lancer dans le public et ceux qui s'y adonnaient étaient assurés de rencontrer la faveur des dames dans les salons du dix-septième siècle: on accueillait ces accusations avec des sourires entendus ! Les écrivains se chargèrent de propager la légende, si bien que beaucoup, même parmi nos gens cultivés, rougissent en parlant de l'origine de leurs aïeules. Il est bien plus facile d'allumer un incendie que de l'éteindre quand le vent s'est chargé de le propager; il en est ainsi pour les rumeurs qui circulent à voix basse puis à la fin au grand jour.

La légende des filles de vie colonisatrices du Canada remonte aux débuts de la colonie; de fait avec Roberval et La Roche s'embarquèrent des vagabonds et des femmes vicieuses: ces établissements étaient voués à un échec: il ne faut pas s'en étonner et c'est heureux pour la réputation de nos premiers colons. Après ces premiers échecs, la France a changé sa politique de peuplement et de colonisation du Canada: la nouvelle compagnie de la Nouvelle-France reçut la propriété du pays et le

97 Benjamin Sulte, Les Canadiens-Français et les Sauvages, dans BRH (1898), IV: 360-363.

${ }_{98}$ Gustave Lanctot, Filles de Joie ou Filles du Roi (Montréal, 1952), 9. 
monopole des fourrures contre l'engagement de le peupler et de l'évangéliser. ${ }^{99}$ Ce n'est qu'en 1634 que se produira le premier mouvement de véritable colonisation du territoire canadien. ${ }^{100}$ En France, on continue de croire que le Canada est une colonie pénale, c'est pourquoi les missionnaires protestent:

On nous a dit (en 1641) qu'il courait un bruit dans Paris, qu'on avait mené en Canada un vaisseau tout chargé de filles dont la vertu n'avait l'approbation d'aucun docteur (...) C'est un faux bruit, dit le Père Le Jeune. J'ai vu tous les vaisseaux: pas un n'était chargé de cette marchandise. ${ }^{1}$

En 1654, le Père Le Mercier, au Canada depuis 1635, ajoute un précieux témoignage en disant que la reine Anne d'Autriche:

envoya ce Printemps dernier quelque nombre de filles fort honnestes, tirées de maisons d'honneur. On n'en reçoit point d'autres dans cette nouvelle peuplade. ${ }^{2}$

En 1658, un autre témoignage, d'un laïc cette fois, vient confirmer les précédents; il s'agit du gouverneur d'Argenson qui écrit:

Il faut que je vous dise une chose qui vous divertira. C'est un jugement que j'ai rendu contre un marchand de La Rochelle appelé Péron: il a esté assez insolent que de nous envoyer en ce pays une fille débauchée et actuellement grosse et qu'il scavait estre en cet état. Je l'ay condamné à la ramener à La Rochelle à touts les dépends qu'il en pouvoit avoir fait et ceux qu'avoit fait celuy à qui il l'avoit donnée en service et en 150 livres d'amende, dont le tiers, je le fais donner à l'hospital de Kébec. Cela remettra nostre pays en réputation que l'on confond avec les Iles Saint-Christophe et empeschera les marchands de se charger de ce bétail. ${ }^{3}$

Le témoignage d'un gouverneur a une grande valeur; il qualifie d'insolence le fait d'amener une fille de mauvaise vie au Canada et il punit sévèrement le coupable, car il veut en

99 Edits et Ordonnances, I: 5-11.

100 Lanctot, op. cit., 41.

1 Ibid., 45.

2 Ibid., $50 \mathrm{~s}$.

3 Lettre d'Argenson, 24 octobre 1658, citée dans Lanctot, op. cit., 51. 
même temps faire une leçon aux marchands qui auraient la fantaisie d'imiter ce geste. Un autre personnage important vient corroborer les dires du gouverneur; c'est Pierre Boucher qui plaide auprès du roi la cause de la Nouvelle-France; en 1661, il dit, en parlant de la rumeur des filles de vie:

Il n'est pas vray qu'il vient icy de ces sortes de filles. Ceux qui en parlent de la façon se sont grandement mépris, et on a pris les Isles de Saint-Christophe et de la Martinique pour la NouvelleFrance: si il en vient icy, on ne les connoist pour telles: car avant que de les embarquer, il faut qu'il y aie quelques-uns de leurs parens ou amis qui assurent qu'elles ont toujours esté sages: si par hazard, il s'en trouve quelques-unes de celles qui viennent, qui soient décriées, ou que pendant la traversée elles ayent eu le bruit de se mal comporter, on les renvoye en France. ${ }^{4}$

Pendant la période qui couvre les années 1634 à 1662, il est venu au Canada 228 filles non accompagnées de leurs parents. ${ }^{5}$ Plusieurs d'entre elles ont été recrutées par Jeanne Mance, M. de La Dauversière, des religieuses, Marguerite Bourgeois, et la plupart par des associations religieuses ou hospitalières. Les jeunes émigrantes, qui venaient seules, le faisaient à la demande de parents déjà établis et elles devaient fournir des certificats de sage conduite. Rendues au pays, même les femmes pouvaient être déportées si elles se conduisaient mal; tel est le cas de «deux vilaines » qui avaient manqué à l'honnêteté: elles furent aussi renvoyées en France. ${ }^{6}$

L'émigration féminine pour cette première période n'a donc pas mérité la réputation qu'on lui a faite; les témoignages précis des missionnaires et des gouverneurs sont là pour le prouver, et ils annulent les propos désobligeants des colporteurs d'histoires malpropres, qui ont induit en erreur leurs contemporains, peu au courant de la géographie américaine. De fait, les Antilles et les Iles ont accepté une émigration tarée: ceci est dû au manque de scrupule des capitaines et des agents qui ont

\footnotetext{
4 Lanctot, op. cit., 53.

5 Ibid., 76.

- Relations des Jésuites, 1654 : 31.
} 
amené des filles de mauvaise vie, pour les revendre à la population masculine, privée de femmes. ${ }^{7}$ La légende, née d'une confusion géographique, a malheureusement persisté, malgré les témoignages non équivoques des témoins oculaires bien qualifiés. $\mathrm{Au}$ regard de l'histoire, cette rumeur est évidemment fausse.

A partir de 1663, la Nouvelle-France passe sous l'administration royale: Louis XIV reprend sous son autorité le territoire et l'administration de la colonie. ${ }^{8}$ Des colons sont envoyés au Canada et on augmente ainsi le nombre des célibataires; pour remédier à ce problème, il fallait aussi recruter des épouseuses ; c'est pendant les années 1663 à 1673 que se formèrent les contingents de filles à marier appelées filles du roi. Le roi payait les frais de recrutement, de voyage et de dotation. Durant cette période, il est passé au Canada 961 filles à marier. ${ }^{9}$ Pour le recrutement, plusieurs modes furent mis en pratique: au début ce furent des enrôleurs à gage, des marchands, puis on s'adressa aux curés et surtout à l'Hôpital Général qui était un hospice et un orphelinat; il faut remarquer que toutes les filles du roi vinrent librement au Canada pour s'assurer un avenir: comme le dit Colbert: "elles sont bien aises d'y passer pour estre mariées et s'y establir ».10 Il faut noter que plus du tiers des filles du roi venaient de l'Hôpital Général, c'est une garantie, puisque dit Lanctot:

Les pensionnaires entraient là dès leur tendre enfance et recevait, au cours de dix ou quinze ans, une excellente formation, fort supérieure à celles des filles de la campagne et même du menu peuple des villes. ${ }^{11}$

De plus une soixantaine de filles étaient « de petite noblesse et de bonne bourgeoisie ». ${ }^{12}$ Pour les autres, nous savons qu'elles viennent de milieux ouvriers et souvent de la campagne. A côté de cette émigration choisie pour le Canada, le peuplement rapide des Antilles nécessitait une émigration en masse; la demande

7 Lanctot, op. cit., 97.

8 Edits et Ordonnances, I: 31s.

9 Lanctot, op. cit., 113.

10 Talon à Colbert, 10 octobre 1665, dans RAPQ (1930-1931) : 39.

11 Lanctot, op. cit., 122. 
était tellement forte qu'on faisait la traite des émigrantes, accordées aux plus offrants. Encore là, l'ignorance géographique a fait confondre le Canada avec les Iles. Heureusement, en ce qui concerne notre pays, la correspondance des intendants nous donne de précieux renseignements. En 1667, Talon écrivait à Colbert:

On a fait entendre à la plus part en france que le Roy leur faisoit plus de cent escus de mariage, particulièrement à celles qui sont de quelque naissance et qui se trouvent au nombre de quinze ou vingt, plusieurs bien Demoiselles et assez bien eslevées. ${ }^{13}$

Les filles du roi trouvent facilement à se marier, car elles sont vraiment choisies, selon le désir de Talon:

Toutes les filles venues cette année sont mariées a quinze préz que j'ay fait distribuer dans des familles connues en attendant que les soldats qui les demandent ayent formé quelques establissemens et acquis de quoy les nourir. (...) Il seroit bon de recommander fortement que celles qui seront destinées pour ce pays ne soient aucunement disgraciées de la nature, quelles n'ayent rien de rebuttant a l'exterieur, qu'elles soient saines et fortes pour le travail de Campagne, ou du moins qu'elles ayent quelque industrie pour les ouvrages de main, j'en escris dans ce sens a $\mathrm{M}^{\mathrm{rs}}$ les directeurs. Trois ou quatre filles de naissance et distinguées par la qualité serviroient peut estre utilement a lier par le mariage des officiers qui ne tiennent au pays que par les appointemens et l'emolument de leurs terres, et qui par la disproportion des conditions ne s'engagent pas d'avantage. Les filles envoyées L'an passé sont mariées, et presque touttes ou sont grosses ou ont eu des enfans, marque de la fécondité de ce pays. (....) Si le Roy fait passer d'autres filles ou femmes veuves de l'ancienne en la nouvelle france, Il est bon de les faire accompagner d'un certificat de leur curé ou du juge du lieu de leur demeure qui fasse connoistre qu'elles sont libres et en estat d'estre mariées, sans quoy les Ecclésiastiques d'Icy

12 Ibid., 120.

13 Talon à Colbert, 27 octobre 1667, dans RAPQ, (1930-1931) : 81. 
font difficulté de leur conferer ce sacrement, a la vérité ce n'est pas sans raison, deux ou trois doubles mariages s'estant icy reconnûs, on pourroit prendre la mesme précaution, pour les hommes veuf. Et cela devroit estre du soin de ceux qui seront chargez des passagers. ${ }^{14}$

Le ministre Colbert répond à ces demandes :

A l'egard de celles qui passeront cette année, j'ay donné les ordres necessaires pour les choisir saines et fortes, et de la qualité que vous les demandez, et je doibs vous dire que le Roy a accordé une gratification de 60011 . à la dam ${ }^{\text {le }}$ Estienne, en consideration du soin que vous me marquez qu'elle a pris de la conduite des autres. J'ay aussy donné ordre de vous envoyer des certificats des lieux où lesd. filles seront prises, qui feront connoistre qu'elles sont libres et en estat de se marier sans difficulté. ${ }^{15}$

Les filles du roi étaient choisies avec soin et elles étaient sous la surveillance de personnes responsables. Le seul reproche qu'on pouvait leur faire c'était le manque de robustesse de certaines émigrantes des villes ... La correspondance du ministre et de l'intendant nous montre que l'on choisissait les émigrantes dans les orphelinats ou les paroisses, souvent avec la collaboration des curés. Au début on exigeait un certificat de bonne conduite, et plus tard l'aptitude au mariage; les candidates devaient être saines physiquement et moralement et elles étaient accompagnées de conductrices de réputation. M. Lanctot conclut:

On exerçait une telle attention dans le choix des filles à marier que, pendant cette période de onze ans que dura cette émigration, on ne peut relever, dans aucun document de l'époque, une seule plainte contre la moralité ou la conduite subséquente des émigrantes. ${ }^{16}$

14 Talon à Colbert, 10 novembre 1670, dans RAPQ, (1930-1931): 125s. 15 Colbert à Talon, 11 février 1671, dans RAPQ, (1930-1931): 144s. 16 Lanctot, op. cit., 225. 
Un autre témoignage précieux est celui du P. Charlevoix:

On avait même apporté une très grande attention au choix de ceux qui s'étoient présentés pour aller s'établir dans la Nouvelle-France, et il n'est pas vrai que les Filles qu'on y envoya, de tems en tems pour les marier avec les nouveaux Habitants ayent été prises dans des lieux suspects, comme quelques Voyageurs peu instruit l'ont avancé dans leurs Relations. On eut toujours soin de s'assurer de leur conduite, avant que de les embarquer, et celle qu'on leur a vu tenir dans le pays est une preuve qu'on y avait réussi. ${ }^{17}$

Après tous ces témoignages, nous devons conclure que le Canada n'a jamais reçu de groupes de filles de joie, bien plus on n'a jamais accepté l'entrée de femmes suspectes ou de mœurs douteuses. L'immigration de cette époque, loin d'être tarée, est des plus saines et des mieux choisies et les émigrantes valeureuses méritent bien le nom de «filles du roi ».

Selon la coutume, une fille en se mariant apportait des biens à son mari : c'était la dot. D'après M. Roy :

$\mathrm{Au}$ pays des ancêtres, une fille sans dot presque toujours coiffait sainte Catherine. La demoiselle avait beau avoir la beauté et toutes les qualités imaginables, elle n'était point recherchée. C'est que le paysan français est beaucoup plus intéressé que l'habitant canadien. Au Canada, même dans les premières années du régime français, un habitant n'épousait pas une fille pour sa dot car elle n'en avait pas. Il la recherchait pour ses solides qualités, sa santé, son esprit de travail. ${ }^{18}$

Ces remarques sont flatteuses pour nos ancêtres, mais elles sont certainement exagérées; de fait, nos aïeules avaient une dot, parfois minime, mais souvent intéressante, car l'argent avait beaucoup de valeur. Ainsi les filles du roi elles-mêmes recevaient une dot royale, comme le dit l'intendant Talon:

Pour advancer le mariage de ces filles je leur ay fait donner ainsy que j'ay accoutumé de faire, outre quelques subsistances, La somme de Cinquante

17 Ibid., 195.

18 Les Cahiers des Dix, IV : 83. 
Livres monnoye de Canada en denrées propres a leur mesnage. ${ }^{19}$

Bien plus le roi voit aussi, à la demande de l'intendant, à doter plus généreusement certaines filles de familles qui par pauvreté ne peuvent trouver à se marier :

Elle (sa majesté) a encore ordonné six mil livres p. appliquer a marier un nomb. de Demoyselles estant $\mathrm{d}^{\mathrm{s}}$ le Pays qui a cause de la pauvreté de leurs familles ne trouvent $\mathrm{p}$. à se pourvoir. ${ }^{20}$

En 1711, le roi envoie encore de l'argent pour encourager les mariages:

Sa Majesté a rétabli la gratification de 3,000 livres pour encourager les mariages. Ce fonds sera employé annuellement à doter soixante filles, ainsi qu'il est indiqué sur l'état des charges. ${ }^{21}$

Ces dons du roi sont très souvent un surplus ajouté à la dot; c'est ainsi que $\mathrm{M}$. Lanctot note:

Ainsi, sur deux cent cinquante contrats de mariage, de l'étude Becquet, pour 1669, 1670 et 1671, on relève soixante-et-six dots de deux cents livres, cent dix-neuf de trois cents, vingt-sept de quatre cents, et plusieurs de cinq cents, six cents et huit cents, avec une de mille livres. ${ }^{22}$

Comme nous pouvons le constater, dans beaucoup de cas, la dot représente un montant assez considérable; parfois c'est une dot en nature; tel est le cas pour le contrat de mariage de Jean Brière:

l'épousée apporte en dot: des assiettes d'étain commun, des cuillères et des fourchettes d'étain fin, un poêlon de cuivre jaune, un fer à flasquer, des couvertes de poil de chèvre, des jupons de camelot, deux habits d'étamine, une coiffe de taffetas noir, des jupons de futaine et de toile de Hollande, des bas de St-Maxant, un corset de futaine et un de carisé, un cor balainé, une jupe d'étamine anglaise, une couverture de poil de chien etc. ${ }^{23}$

19 Talon à Colbert, 10 novembre 1670, dans RAPQ, (1930-1931) : 125.

20 Colbert à Talon, 20 février 1668, Ibid., 92.

21 Le roi à Vaudreuil, 7 juillet 1711, dans RAPQ, (1940-1941) : 427.

22 Lanctot, op. cit., 202.

23 Cloutier, op. cit., I: 200. 
Ferland nous rapporte un cas datant de 1647 :

Magdelaine Boucher, qui se maria avec Urbain Baudry dit Lamarche, était fille de Gaspar et sœur de Pierre Boucher des Trois-Rivières. Voici ce qu'elle apportait à son époux par son contrat de mariage: Deux cents franes en argent; quatre draps; deux nappes; six serviettes de toile et de chanvre; un matelas et une couverture; deux plats; six cuillers et six assiettes d'étain ; une marmite et une chaudière; une table et deux formes; une huche à boulanger; un coffre fermant à clef; une vache; deux cochons, mâle et femelle. A la mariée les parents donnaient un habit, selon sa qualité, et du linge à sa discrétion. ${ }^{24}$

Les dots varient beaucoup en quantité et elles revêtent les formes les plus variées; en voici quelques exemples : le 19 janvier 1664, Alexandre Raoul et Marie Desrosiers passaient leur contrat de mariage, la dot de l'épouse était de « 300 livres tournois $\gg^{25}$ Le 26 novembre 1663, c'était le tour de François Chorel et d'Anne Aubuchon: la dot était de «six cents livres tournois, une concession de vingt-cinq arpents aux Trois-Rivières, pendant six ans et une maison dans la même ville, sise en la place des Sauvages, pendant deux ans $\gg .^{26}$ Renée Chorel s'allia le 17 février 1692 à Jacques le Picard, la dot de «quatre mille livres lui fut payée en $1694 \gg .^{27}$ Le 3 novembre 1644, Martin Prevost épousait Marie Olivier, une sauvagesse; le parrain de cette dernière, $M$. de Puiseaux, « lui fournit une dot de cinq cents livres ».28 La jeune Anne Baillargeon fut enlevée par les Iroquois; après neuf ans de vie nomade, elle revint à la civilisation; c'est alors que «Tracy lui donna cinquante écus pour se marier $\gg .^{29}$

Ainsi les dots existaient toujours sous le Régime français; elles étaient parfois minimes mais souvent elles représentaient des valeurs considérables et de nature à attirer les jeunes gens

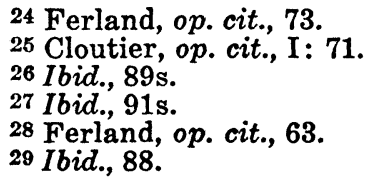


vers le mariage. La dot était fixée à l'occasion du contrat de mariage. Un des plus anciens qui nous soient parvenus porte la date du seize juillet 1636. Voici ce qu'en dit Ferland:

Guion, quoique simple maçon, était l'homme de lettres de Beauport. Un contrat dressé par lui pour le mariage de Robert Drouin, de la paroisse Du Pin, chatellenie de Mortagne au Perche, avec Anne Cloustier, est bien écrit, et l'orthographe y est respectée. Ce contrat de mariage le plus ancien peut-être qui se soit conservé, puisque la date est du seize juillet 1636, est signé des membres de la famille Giffard, de François Bellenger, de Noël Langlois; les autres témoins ont apposé leurs marques, parmi lesquelles prime la hache de Zacharie Cloustier. ${ }^{30}$

(à suivre)

PAUL-ANDRÉ LECLERC, ptre, Licencié ès-lettres, Univ. Laval, Collège de Ste-Anne-de-la-Pocatière, P.Q.

30 Ibid., 65.

\section{ARTICLES A PARAITRE DANS NOS PROCHAINES LIVRAISONS :}

LUC LACOURCIÈRE, «Ph.-Aubert de Gaspé, témoin de la tradition 》.

L. JoRE, «Un Canadien, gouverneur du Sénégal, Louis Le Gardeur de Repentigny, 1721-1786».

ADRIEn Pouliot, s.j.: «L'exploit du Long-Sault, ses résultats 》.

Antanio Drolet : «La bibliothèque du Collège de Québec, (16321800)».

ROLAND LAMONTAGNE : "La personnalité de Roland-Michel Barrin de La Galissonière ».

ROBERT Sylvain, e.c.: «Le 9 juin 1853 à Montréal ». et quelques autres. 